\title{
THE DIVERSITY OF FLOWER COLOUR: HOW AND WHY?
}

\author{
B.J. GLOVER \\ Department of Plant Sciences, University of Cambridge, UK.
}

\begin{abstract}
The diversity of flower colour has astonished artists, gardeners and scientists for centuries. Flowers generate colour by reflecting only a subset of the wavelengths which make up white light, resulting in a coloured appearance. This is achieved either through the use of chemical pigments which absorb certain wavelengths, or by the use of structures which reflect only certain wavelengths. Chemical colour has been well studied in plants, and the three major pigment groups are flavonoids, carotenoids and betalains. Spatial and temporal regulation of the synthesis of these pigments gives pattern and depth of colour to the flower. Combinations of pigments can result in variations in final flower colour, while the addition of metal ions and the alteration of cell pH can also influence the final wavelengths absorbed by pigments. Focussing light into the pigment-containing regions of the cell, using specialised cell shapes, also influences intensity of flower colour. Structural colour, including iridescence, is produced independently of pigment colour, and can overlay it. Flower colour itself is viewed as an advertisement to attract pollinating animals to the rewards (usually nectar) contained within the flower. This article concludes with an analysis of the long-running debate over whether specific flower colours attract specific pollinators, or whether all colours are simply different ways of attracting a wide variety of animals. Keywords: anthocyanin, betalain, carotenoid, flower, iridescence, petal, pigment, pollination, structural colour.
\end{abstract}

\section{INTRODUCTION}

The bright colours of flowers are primarily a signal to attract pollinating insects by making the floral tissue stand out against a background of vegetation. This argument is supported by modern analysis of insect visual acuity, which indicates that vegetation is visually very similar to bark, soil and stone from an insect's point of view. All these materials weakly reflect light across the whole range of an insect's visual spectrum. Leaves differ from the rest only in absorbing red light, but since red is at the very periphery of the visual spectrum for most insects, this makes little difference [1].

Biological colours can be produced in two different ways. Many animals produce 'structural' colours, caused by the refraction of light from complex physical surfaces. Although there is some evidence that plants can use structural colour too, they primarily produce colour by synthesising pigments, which absorb subsets of the visible spectrum, reflecting back only what they do not absorb and causing the tissue to be perceived as the reflected colours. Chlorophyll absorbs light in both the red and blue parts of the spectrum, reflecting only green light, and causes leaves to appear green to us. Similarly, a flower that we perceive as red contains pigments, which absorb yellow, green and blue light, leaving red light as the only wavelength visible to us which is reflected.

\section{CHEMICAL COLOUR IN FLOWERS}

Chemical colour is produced through the absorption of light by pigments. Plant pigments can be divided into three chemical classes: the flavonoids, the betalains and the carotenoids. The flavonoids are the major floral pigments, and give rise to ivory and cream colours (through pigment types called flavonols and flavones), yellow and orange colours (through aurones and chalcones) and the redpink-purple-blue range (the anthocyanins, Fig. 1a). The betalains are a group of pigments found exclusively in the Angiosperm order Caryophyllales, and nowhere else in the plant kingdom. They give the red colour to beetroot and also to some flowers (Fig. 1c). The carotenoids are much more widespread, although less significant as floral pigments than the flavonoids. Carotenoids give yellow and orange colour to flowers (Fig. 1b).

(C) 2009 WIT Press, www.witpress.com

ISSN: 1755-7437 (paper format), ISSN: 1755-7445 (online), http://journals.witpress.com DOI: 10.2495/DNE-V4-N3-211-218 

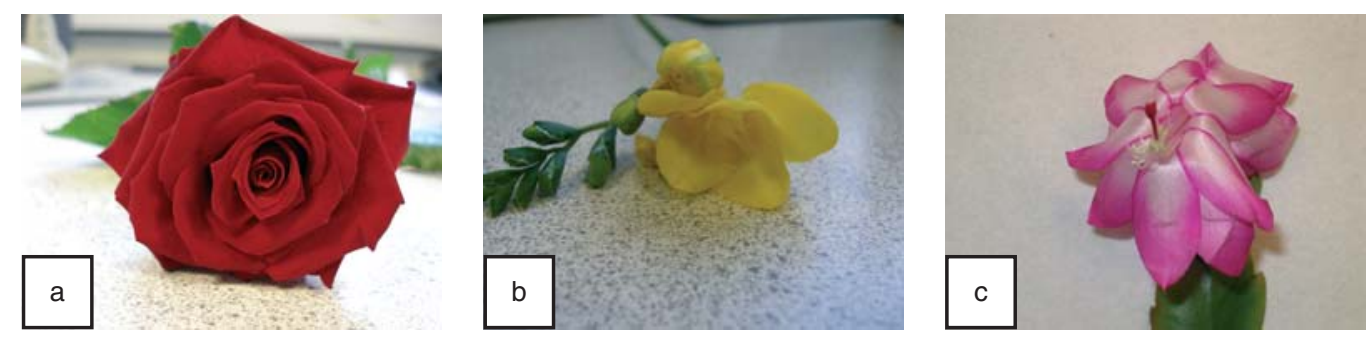

Figure 1: Flower colour produced by (a) anthocyanins in rose, (b) carotenoids in Freesia, (c) betalains in Christmas cactus.

\subsection{Flavonoids}

The flavonoids are a group of phenolic compounds in which two six-carbon rings are linked by a three-carbon unit. Flavonoids are water soluble and accumulate in the vacuoles of higher plant cells. They play a number of roles in plant physiology and development. Flavonoids may be involved in defence against pathogens and predators, are a component of the legume-Rhizobium signalling cassette, are required for correct pollen development and pollen tube growth, protect sensitive tissues from ultraviolet radiation, and act as antioxidants and metal chelators.

Flavonoid synthesis has been characterised in some detail, both from a biochemical perspective and from a molecular genetic one (reviewed by Martin and Gerats [2]). The most detailed studies have used the petals of Antirrhinum and Petunia as models. Antirrhinum flowers normally produce a magenta anthocyanin, called cyanidin. Petunia flowers produce a purple anthocyanin, delphinidin. Since the blueness of the anthocyanin is determined by the degree of hydroxylation of the B ring, in theory, a plant can always hydroxylate the molecule less, and thus make less blue anthocyanins, but it will not necessarily have the enzymes to hydroxylate it more, and so cannot make bluer anthocyanins. In fact, investigation of this hypothesis in Petunia has revealed that the enzymes of the biosynthetic pathway have a greater efficiency for conversion of their usual substrates, and cannot therefore easily produce orange pelargonidins from less hydroxylated substrates [3].

The first committed step of flavonoid synthesis is the condensation of three acetate units and a hydroxycinnamic acid unit to produce chalcone, the key intermediate in the synthesis of all flavonoids [2]. Chalcone itself is usually yellow or orange, and can be used as a pigment in its own right. It may also be converted into a yellow aurone, the pigment which provides the bright yellow colour to many Compositae flowers, such as Dahlia [4]. Usually, however, chalcone is modified to a colourless flavanone, and flavanone then feeds into one of three further pathways. Flavanone may be directly converted into flavones, which vary in colour from very pale to bright yellow, depending on their degree of hydroxylation. Alternatively, flavanone can be converted into dihydroflavonol, which can then be modified by flavonol synthase to various flavonols. The flavonols are usually colourless, but act as co-pigments, stabilising and modifying the colour of other pigment molecules. Alternatively, dihydroflavonols may be modified through a number of steps to make anthocyanins. Anthocyanins provide a number of different colours, depending on several factors. They may range from orange/brick red (known as pelargonidins), red/magenta (cyanidins) to purple/blue (delphinidins), with increased blueness determined by an increase in hydroxyl groups. The two main classes of glycosides also affect the blueness of the molecule. Methylation of an anthocyanin tends to shift its colour towards red, compared to bluer unmethylated molecules. 
Anthocyanin is transported across the vacuolar membrane into the vacuole, where it is stored, by the glutathione pump [5]. The pigment is first conjugated with glutathione, by the enzyme glutathione S-transferase.

\subsection{Carotenoids}

Carotenoids are amongst the most widespread pigments in the natural world. In plants, they play important roles in photosynthesis, where they act as accessory light harvesting pigments and as photo-protectants [6], as well as acting as floral and fruit pigments. In mammals they are the precursors for vitamin A synthesis, and in fish they are essential for phototropic responses [7]. The carotenoids are a family of isoprenoid derivatives. Isoprenoids themselves are lipid molecules, with an estimated 22,000 different types known. They have essential roles as membrane sterols, components of chlorophyll, cytokinins, abscisic acid and a variety of roles in plant secondary metabolism. They are usually classified by the number of carbon atoms they contain. The carotenoid family contains conjugated polyene molecules, composed of 40 carbon atoms. Carotenoid hydrocarbons are known as carotenes, and include pigments such as zeaxanthin. The addition of oxygen to these molecules creates the oxygenated carotenoids, or xanthophylls, which are also important pigments.

The absorption spectrum of a particular carotenoid molecule is determined by the conjugated polyene system and additional structural features. Each conjugated double bond increases the wavelength of maximum absorption by 7-35 nm [7]. Thus, deep orange flowers may contain unoxidised lycopene, orange flowers contain $\beta$-carotene, and yellow flowers often contain highly oxidised xanthophylls.

\subsection{Betalains}

The betalains have been less well studied than the carotenoids and anthocyanins. They replace anthocyanins as the colouration in flowers and fruit of the order Carophyllales [8]. Common betalainpigmented plants include beetroot, the petals of the Christmas cactus and the brightly coloured bracts of Bougainvillea.

The betalains are water-soluble nitrogen-containing pigments, and come in two main colour groups. The betacyanins are red to purple, while the betaxanthins are yellow. The early and late steps of betalain synthesis are catalysed by enzymes, but the vast majority of intermediate reactions in the synthetic pathway occur spontaneously [8].

\section{MODIFYING CHEMICAL COLOUR IN FLOWERS}

\subsection{Regulating pigment synthesis}

Since the types of pigments produced has such a range of effects on flower colour it is hardly surprising that the pigmentation pathways should be under tight regulatory control. This regulation is both spatial and temporal, generating colour patterns on almost all flowers (Fig. 2a), and in some species changing the colours of petals during their development. For example, the flowers of Viola cornuta cultivar "Yesterday, Today and Tomorrow" change from white to pale pink to purple over the space of 5-8 days. This change in colour has been shown to be the result of a steady increase in anthocyanin production over the time period. While such a colour change may seem extraordinary, the fact that it is initiated by pollination (without which the petals remain white) suggests that plant growth regulator-mediated signals from germinating pollen tubes trigger the change in anthocyanin regulation, perhaps to change the attractiveness or visibility of the flower to potential pollinators [9]. 

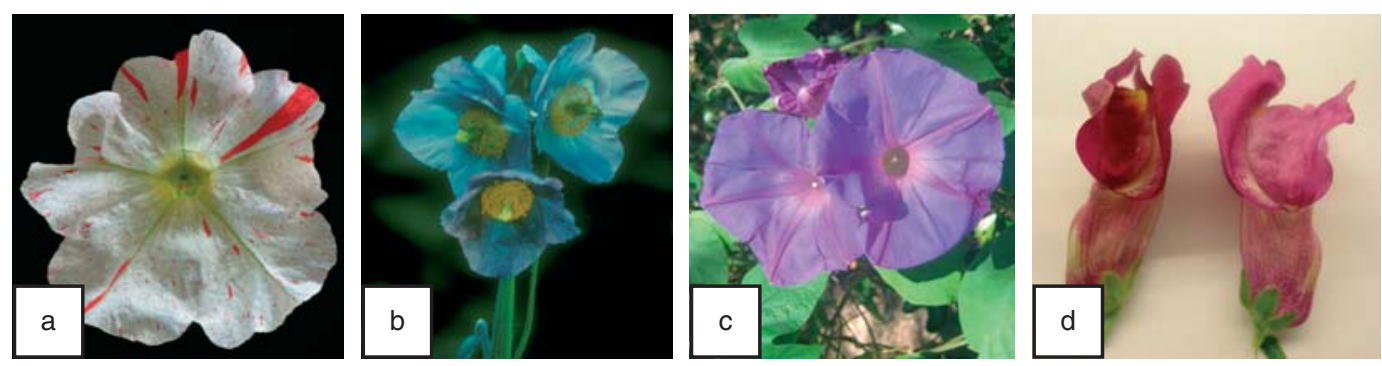

Figure 2: Final flower colour is controlled by (a) spatial regulation of pigment synthesis in Petunia hybrida, (b) metal ions in Meconopsis, (c) pH in Ipomoea, (d) petal cell shape in Antirrhinum majus (left, flower with conical petal epidermal cells, right, flower with flat petal epidermal cells).

It has been shown that the activities of the genes encoding the enzymes of anthocyanin biosynthesis are predominantly regulated at the transcriptional level. It can be inferred from this, that the majority of petal pigmentation patterns are specified by the expression patterns of regulatory genes which control the activity of the biosynthetic genes [3]. By convention, anthocyanin regulatory loci are divided into those which specifically control anthocyanin deposition (by regulating 'late' biosynthetic genes, some way down the pathway), and those which control the synthesis of other flavonoids (by regulating 'early' biosynthetic genes). A number of genes have been shown to be important in both pathways, and three key groups of proteins have been shown to be involved. The most important of these are transcriptional activators of the MYB and basic helix-loop helix families (reviewed by Martin et al. [10]).

\subsection{Metal ions}

Interactions between floral pigments and metal ions can also alter the final colour of the petals (Fig. 2b). For example, the bright blue colour of cornflowers stems from an interaction between the purple anthocyanin, delphinidin and molecules of the metal iron, absorbed by the plant's roots from the soil. The combination of the pigment with the metal results in a molecule that gives the flower, a very bright blue colour. Another good example of this sort of interaction is the variable colour of the flowers of Hydrangea. Hydrangea flowers are blue if there is aluminium in the soil, as aluminium and delphinidin form a very stable, very blue complex. If there is less aluminium available in the soil, and more molybdenum, then the flowers appear pink instead. The same pigment interacts with molybdenum ions and changes to a light pink colour. The presence of this changeable pigment in Hydrangea is exploited by gardeners, who water the soil around their plants with a solution containing the appropriate ion to generate the final flower colour of their choice.

\section{$3.3 \mathrm{pH}$}

The $\mathrm{pH}$ of petal cells can also affect the final colour of the flower, as $\mathrm{pH}$ determines anthocyanin structure and absorption spectrum. For example, the light blue petals of Ipomoea tricolor, Morning Glory, owe their colour to the effect of a high petal $\mathrm{pH}$ on their anthocyanin (Fig. 2c). The closed buds of these flowers are purplish red and their cells have a $\mathrm{pH}$ of 6.6. However, when the flowers open petal cell $\mathrm{pH}$ increases to 7.7, and the pigment changes colour to sky blue [11]. Yoshida et al. [12] 
discovered that the increased $\mathrm{pH}$ is due to active transport of $\mathrm{Na}^{+}$and/or $\mathrm{K}^{+}$from the cytosol to the vacuoles. The ability of vacuolar $\mathrm{pH}$, controlled by membrane transporters, to alter flower colour without any change in the types of pigment produced, gives plants the flexibility to alter their final petal colour after pigments have been made. Stewart et al. [13] observed that the colours of several wild flower species became bluer as they aged, correlated with an increase in petal $\mathrm{pH}$.

\subsection{Focussing light into pigment}

A very subtle way in which the colour of a petal can be enhanced is by focusing of light into pigmented regions. Kay et al. [14] proposed that conical-papillate shaped epidermal cells increased the amount of light absorbed by the pigments in flowers, enhancing the perceived colour of the petal.

The mixta mutant of Antirrhinum fails to develop conical-papillate petal cells and instead has flat petal cells. The significance of the conical-papillate cells in enhancing colour is shown by the fact that the mixta mutant was originally identified in a screen of mutagenised plants, because it was paler in colour than wild type flowers (Fig. 2d). The mutant petal also has a matt texture, unlike the velvety sparkle of the wild type petal [15].

By comparing the ability of epidermal cells to focus light in the wild-type and mixta mutant lines, conical-papillate cells have been shown to enhance visible pigmentation. Conical-papillate cells focus light approximately twice as well as the mutant flat cells, and they focus it into the region of the epidermis where the pigment is contained [16]. The wild-type Antirrhinum petals reflected significantly less light away from the flower than mixta mutant petals did, and absorbed significantly more light. These differences can be attributed to the focusing of the light onto the light-absorbing pigments in the epidermal cells, and to the reduction in reflection of light at low angles of incidence, resulting in the greater depth of colour of wild-type conical-celled flowers [16].

\section{STRUCTURAL COLOUR IN FLOWERS}

Structural colours have been very poorly studied in plants, and are generally thought of as an animal phenomenon. However, a recent report suggests that they might be surprisingly widespread in plants, just mainly visible in the ultraviolet region of the spectrum. This would make them clearly apparent to most pollinating animals, but invisible to the human eye.

Whitney et al. [17] observed iridescence over the red pigmented patch at the base of the Hibiscus trionum petal. Iridescence is the change in hue of a surface when viewed from different angles, and can only be generated by structural (not pigment-based) methods. They analysed the structure of the petal, and found that the epidermal cells in the iridescent region, where overlain with long thin stripes of cuticle. These cuticular striations were shown to be of the same frequency and amplitude as the diffraction grating on a compact disk (CD), and to generate iridescence through the same interference with light reflection as shown by a CD. Whitney et al. [17] further demonstrated that bumblebees could see the iridescence arising from the diffraction gratings, and could be trained to associate it with a nectar reward.

Cuticular striations are very commonly found on plant epidermal surfaces. Their ability to function as diffraction gratings will be strongly dependent on the extent to which they are ordered. However, preliminary analyses have identified suitable striations in 10 plant families to date, as well as in many garden varieties of tulip (Whitney and Glover, unpublished). Although very few of these flowers look iridescent to the human eye, analysis of the diffractive optics of their diffraction gratings indicates that the bulk of the structural colour produced is in the blue and ultraviolet part of the spectrum. This region is highly visible to animal pollinators, especially insects, but not visible to the human eye. It therefore seems likely that many flowers produce structural colours, including 
iridescent ones, through the use of diffraction gratings made from cuticle, but that the structural colours they produce are rarely visible to people.

\section{WHY THE DIVERSITY OF FLOWER COLOUR?}

Colours act as advertisements, enticing animal pollinators to visit flowers for the rewards that they contain. What is less clear is whether particular colours act as advertisements to particular animals. In the rush to find evidence to support Darwin's evolutionary theory, the visually obvious similarities between flower colour and pollinator preferences were seized upon by many authors as clear examples of the consequences of natural selection. It was only in the 1980s that experimental approaches were first taken to assess whether particular floral traits were under selection by pollinators. In 1996, two critical essays were published, both questioning the concept of plant/pollinator specialisation $[18,19])$. Both papers suggested that generalisation, with flowers receiving pollinator service from more than one type of animal, was very frequent in nature. The data now emerging allow us to make initial comments on the utility of the idea that different flower colours acts as advertisements to particular animals.

The literature contains many examples of attempts to assess pollinator discrimination between petals of different colours. In some cases, discrimination is clear, in others, the animals showed no discrimination, and in many cases some animals discriminated while others did not. These experiments indicate that the attraction of multiple pollinators can result in mixed selective pressures, even where one animal shows very clear discrimination. For example, Raphanus raphanistrum, wild radish, has yellow flowers or white flowers, controlled by a single locus. The frequency of the yellow morph varied from $7 \%$ to $60 \%$ in the populations studied by Kay [20], but in all these populations the butterfly Pieris rapae much preferred the yellow form to the white. On the site with $60 \%$ yellow flowers, 307 visits to wild radish flowers by the butterflies were observed - and 306 of the 307 were to yellow flowers. However, honey bees showed no preference for yellow flowers over white, maintaining the polymorphism within the population.

Bradshaw and Schemske [21] provided clear evidence that both bumblebees and hummingbirds distinguish between different coloured forms of Mimulus, using near isogenic lines that would be unlikely to differ in traits other than flower colour itself. Mimulus lewisii is normally pink, as a result of anthocyanin deposition, and is primarily pollinated by bumblebees. M. cardinalis is normally orange/red, as a result of both anthocyanin and carotenoid deposition, and is primarily pollinated by hummingbirds. Bradshaw and Schemske [21] introgressed the YUP locus, responsible for carotenoid deposition, from each species into the other background, through four generations, ensuring $97 \%$ genetic identity between the new lines and their most similar parent. This resulted in orange coloured M. lewisii flowers and deep pink M. cardinalis flowers. Pollinator visits to these flowers were recorded, and revealed that orange-flowered $M$. lewisii received 68-fold more visits from hummingbirds than the wild type pink, but a significant reduction in bumblebee visits. Similarly, the pink-flowered M. cardinalis received 74-fold more visits from bumblebees than the wild type orange (although little reduction in hummingbird visits). These experiments show that both bumblebees and hummingbirds exhibit strong discrimination on the basis of petal colour. The near isogenic nature of the lines used in this study makes it likely, although not certain, that colour is the only significant factor in the choices made by pollinators.

These studies, and many others like them, tell us that animals can discriminate between different flower colours, and often do so. However, it is difficult to extrapolate from this to a view that each individual flower colour is a specialist advertisement to a particular animal. Indeed, most pollinators can learn to associate any colour they can see with food, and so will learn to view the array of flower colours before them as indicating a range of potential food sources. The answer to whether there are 
specialised relationships between different flower colours and different animals then comes down to a question of how frequently plants specialise on a single pollinator species or group, and how frequently they are pollinated by a wide range of animals. Empirical data on the frequency of specialisation in pollination systems are currently in short supply, but it is likely that a good degree of generalisation exists, leading us to conclude that much of the diversity of flower colour is simply due to the many different solutions plants have evolved to the problem of attracting animals by standing out against a green background.

\section{CONCLUSIONS}

The diversity of flower colour is astonishing, especially when compared to the relative uniformity of colour of other plant organs, such as leaves. That diversity is attributable to complex combinations of different pigments, located in different parts of the cell, and regulated in their synthesis both temporally through the life of the flower and spatially among the different floral regions. Pigments are enhanced and modified through the use of metal ions, specific $\mathrm{pH}$ regimes and specialised cell shapes which focus light. They can also be overlain with structural colours which may be iridescent and most frequently reflect wavelengths visible to insects but not to the human eye. This great diversity of flower colour serves as advertising to the enormous variety of animal pollinators, but much more empirical evidence is needed before we can say with confidence whether particular colour regimes attract particular animals, or whether all colours simply serve to make flowers stand out from the surrounding foliage.

\section{REFERENCES}

[1] Kevan, P., Giurfa, M. \& Chittka, L., Why are there so many and so few white flowers? Trends in Plant Science, 1, pp. 280-284, 1996. doi:10.1016/1360-1385(96)20008-1

[2] Martin, C. \& Gerats, T., The control of flower coloration. The Molecular Biology of Flowering, ed. B. Jordan, CAB International: Wallingford, pp. 219-255, 1993.

[3] Mol, J., Grotewold, E. \& Koes, R., How genes paint flowers and seeds. Trends in Plant Science, 3, pp. 212-217, 1998. doi:10.1016/S1360-1385(98)01242-4

[4] Nakayama, T., Enzymology of aurone biosynthesis. Journal of Bioscience and Bioengineering, 94, pp. 487-491, 2002. doi:10.1263/jbb.94.487; doi:10.1016/S1389-1723(02)80184-0

[5] Marrs, K.A., Alfenito, M.R., Lloyd, A.M. \& Walbot, V., A glutathione S-transferase involved in vacuolar transfer encoded by the maize gene Bronze-2. Nature, 375, pp. 397-400, 1995. doi: $10.1038 / 375397 \mathrm{a} 0$

[6] Hirschberg, J., Production of high-value compounds: carotenoids and vitamin E. Current Opinion in Biotechnology, 10, pp. 186-191, 1999. doi:10.1016/S0958-1669(99)80033-0

[7] Goodwin, T.W., The Biochemistry of the Carotenoids, Vol. 1, Chapman and Hall: New York, 1980.

[8] Strack, D., Vogt, T. \& Schliemann, W., Recent advances in betalain research. Phytochemistry, 62, pp. 247-269, 2003. doi:10.1016/S0031-9422(02)00564-2

[9] Farzad, M., Griesbach, R. \& Weiss, M.R., Floral colour change in Viola cornuta L. (Violaceae): a model system to study regulation of anthocyanin production. Plant Science, 162, pp. 225-231, 2002. doi:10.1016/S0168-9452(01)00557-X

[10] Martin, C., Prescott, A., Mackay, S., Bartlett, J. \& Vrijlandt, E., The control of anthocyanin biosynthesis in flowers of Antirrhinum majus. Plant Journal, 1, pp. 37-49, 1991. doi:10.1111/ j.1365-313X.1991.00037.x; doi:10.1046/j.1365-313X.1991.t01-14-00999.x

[11] Yoshida, K., Kondo, T., Okazaki, Y. \& Katou, K., Cause of blue petal colour. Nature, 373, p. 291, 1995. doi:10.1038/373291a0 
[12] Yoshida, K., Kawachi, M., Mori, M., Maeshima, M., Kondo, M., Nishimura, M. \& Kondo, T., The involvement of tonoplast proton pumps and $\mathrm{Na}^{+}\left(\mathrm{K}^{+}\right) / \mathrm{H}^{+}$exchangers in the change of petal colour during flower opening of morning glory, Ipomoea tricolor cv. Heavenly Blue. Plant and Cell Physiology, 46, pp. 407-415, 2005. doi:10.1093/pcp/pci057

[13] Stewart, R.N., Norris, K.H. \& Asen, S., Microspectrophotometric measurement of pH and $\mathrm{pH}$ effect on color of petal epidermal cells. Phytochemistry, 14, pp. 937-942, 1975. doi:10.1016/0031-9422(75)85162-4

[14] Kay, Q.O.N., Daoud, H.S. \& Stirton, C.H., Pigment distribution, light reflection and cell structure in petals. Botanical Journal of the Linnean Society, 83, pp. 57-84, 1981. doi:10.1111/ j.1095-8339.1981.tb00129.x

[15] Noda, K., Glover, B.J., Linstead, P. \& Martin, C., Flower colour intensity depends on specialised cell shape controlled by a MYB-related transcription factor. Nature, 369, pp. 661-664, 1994. doi: $10.1038 / 369661 \mathrm{a} 0$

[16] Gorton, H.L. \& Vogelmann, T.C., Effects of epidermal cell shape and pigmentation on optical properties of Antirrhinum petals at visible and ultraviolet wavelengths. Plant Physiology, 112, pp. 879-888, 1996.

[17] Whitney, H., Kolle, M., Andrew, P., Chittka, L., Steiner, U. \& Glover, B.J., Floral iridescence, produced through diffractive optics, acts as a cue for animal pollinators. Science, $\mathbf{3 2 3}$, pp. 130-133, 2009. doi:10.1126/science.1166256

[18] Herrera, C., Floral traits and plant adaptation to insect pollinators: a devil's advocate approach. Floral Biology, eds D. Lloyd \& S. Barrett, Chapman and Hall: New York, pp. 65-87, 1996.

[19] Waser, N., Chittka, L., Price, M., Williams, N. \& Ollerton, J., Generalization in pollination systems, and why it matters. Ecology, 77, pp. 1043-1060, 1996. doi:10.2307/2265575

[20] Kay, Q.O.N., Preferential pollination of yellow-flowered morphs of Raphanus raphanistrum by Pieris and Ersistralis spp. Nature, 261, pp. 230-232, 1976. doi:10.1038/261230a0

[21] Bradshaw, H. \& Schemske, D., Allele substitution at a flower colour locus produces a pollinator shift in monkeyflowers. Nature, 426, pp. 176-178, 2003. doi:10.1038/nature02106 